\title{
Flambage d'un cylindre à paroi mince, placé dans une enveloppe rigide et soumis à une pression extérieure
}

\author{
Application aux blindages métalliques des puits et galeries
}

\section{Buckling of a thin walled tube fitted in a rigid outer covering and subjected to an external pressure}

\author{
Application to metal linings for wells and tunnels \\ PAR H. BOROT

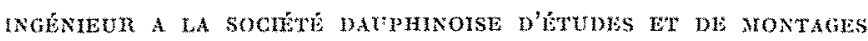

\begin{abstract}
Accidents pronoqués sur aes blindages par des sous-pressions pronenant d'inflitrations d'ean ou d'injections de ciment.

Etude mathèmatique du phénomène de fantbage liè à l'instabilité de la paroi, puis à lat limite élasfique de l'acier.

Application anx blindages métalliques alos puils et galeries. - Graphique donnant les pressions de flambage pour des limites élastiques de 24 et $34 \mathrm{~kg} / \mathrm{mm}^{2}$.

Verifcation experimentale. - Description du materiel el critiques des résultals.

Conclusion.
\end{abstract}

De nombreux aceidents survenus à des blindages métalliques de puits ou galeries, généralement pendant une vidange, ont démontré que toute conduite souterraine bétonnée devait obligatoirement être calculée pour résister au flambage sous l'effet de pressions extérieures. Ces sous-pressions sont provoquées en grande partic par des infiltrations d'eau dans le sol, provenant de sources naturelles ou d'ouvrages hydrauliques.

Actuellement, tout nouveau blindage est sou-

\begin{abstract}
Tregularities in linings catised by underpressure resulting from whiter seepage or injection of cement.

Malhematical study of the buckling phenomenon related to wall insiability and the plastic limit of the stepl.

Application to metal linings for wells and tunnels. - Diagram showing buckling pressures for elastic limits of 24 and $34 \mathrm{~kg} / \mathrm{mm}^{2}$.

Experimental verification. -..- Description of equipment and comments on the resulls.

Conclusion.
\end{abstract}

mis, après son bétonnage, à des injections qui sont destinées, d'une part à combler les vides qui se produisent pendant la mise en place du béton de garnissage, et d'autre part, à lestituer au rocher sa compression initiale nécessaire à la bonne tenue du blindage sous la pression intérieure. Ces injections se font à des pressions assez élevées et on connait quelques cas où le blindage a subi une déformation importante, consécutive à une absence de contrôle sérieux de la pression d'injection. 


\section{I. - ETUDE DU FLAMBAGE}

Notatrons (fig. 1)

$\mathrm{P}=$ pression extérieure;

$\mathrm{E}:=$ module d'élasticité;

$\mathrm{I}=$ module d'inertie $=e^{3 / 12}$;

$n=$ nombre d'ondes;

$\mathrm{R}=$ rayon extérieur du cylindre;

$e=$ épaisseur de la paroi;

$\mathrm{K}=e / \mathrm{R}$;

$t=$ limite élastique de la paroi;

$y_{0}=$ fliche au centre de l'onde $=A B$;

$j=$ jeu entre enveloppe el cylindre pour $\mathrm{P}=0$;

$\mathrm{S}=$ Iongueur d'une demi-onde $\mathrm{B}$ C.

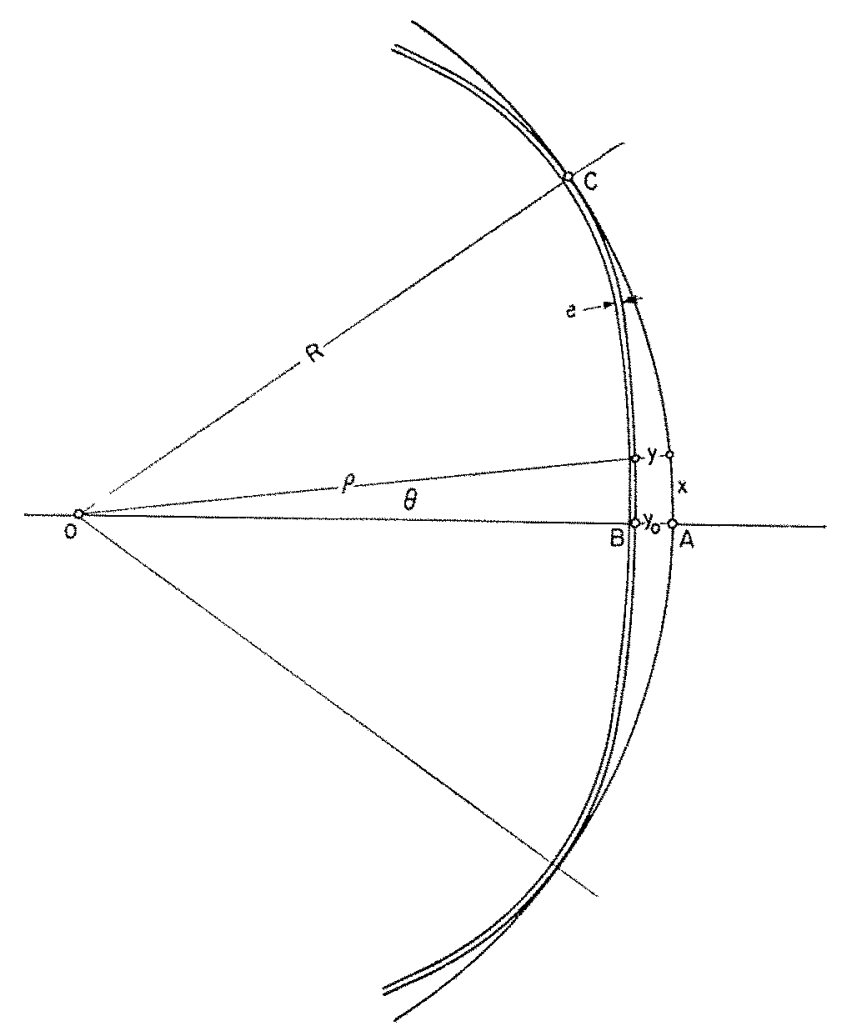

Fik. 1

Nous avons considéré le cas d'un cylindre à paroi mince, sans renfort ni ancrage, placé à l'intérieur d'une enveloppe que nous avons supposée indéformable. Nous avons admis qu'il n'existait aucun vide local important entre le cylindre et l'enveloppe, ce qui revient à dire, pour les blindages de puits et galeries, que les injections ont été faites correctement, et que les points d'injection ont été suffisamment nombreux pour combler tous les creux qui peuvent subsis- ter derrière le blindage après bétonnage. Nous avons d'autre part supposé que la pression extérieure était également répartie tout autour du cylindre.

Notre étude est faite pour un cylindre non précontraint, c'est-à-dire qu'entre l'enveloppe et le cylindre, il existe un jeu de faible valeur. C'est le cas des blindages qui n'ont pas été soumis à une précompression, soit par frettage, soit par injections spéciales.

Certains auteurs ont montré que, le long de la paroi d'un cylindre soumis à une pression extérieure, se forme un certain nombre d'ondes qui dépend des liaisons extérieures du cylindre. Ils ont établi que la pression critique de flambage est donnée par :

$$
\mathrm{P}=\frac{\mathrm{EI}}{\mathrm{R}^{3}}\left(n^{2}-1\right)
$$

dans laquelle $n$ est le nombre d'ondes.

La déformée d'une barre mince à ligne moyenne circulaire est donnće par l'équation différentielle :

$$
\frac{d^{2} y}{d x^{2}}+\frac{y}{\mathrm{R}^{2}}=-\frac{\mathrm{M}}{\mathrm{EI}}
$$

L'équation suivante donne la forme de l'onde de flambage :

$$
y=\frac{y_{0}}{2}\left(1+\cos \frac{n x}{\mathrm{R}}\right)
$$

Nous déduisons la loi des moments tléchissants des équations (2) et (3) :

$$
\mathrm{M}=\frac{\mathrm{E} e^{3} y_{0}}{24 \mathrm{R}^{3}}\left[\left(n^{2}-1\right) \cos \frac{n x}{\mathrm{R}}-1\right]
$$

Le moment fléchissant est maximum au point C, pour $x=(\Pi \mathrm{R} / n)$.

$$
\mathrm{M}_{c}=-\frac{\mathrm{E}^{3} y_{0} n^{2}}{24 \mathrm{R}^{2}}
$$

Au point $\mathrm{C}$, dans les fibres extérieures de la paroi, nous avons :

1. Une contrainte de compression :

$$
t_{c}=\frac{\mathrm{PR}}{e}
$$

2. Une contrainte de flexion :

$$
t_{f}=-\mathrm{M}_{v} \frac{6}{e^{2}}=\frac{\mathrm{E} e y_{0} n^{2}}{4 \mathrm{R}^{2}}
$$


Etudions maintenant les variations de lonsueur de l'onde pendant son enfoncement. La longueur d'une courbe plane, en coordonnces polaires, est donnée par:

$$
S=\int_{\mathrm{R}}^{C}\left[\rho^{2}+\left(\frac{d \rho}{d \theta}\right)^{2}\right]^{1 / 2} d \theta
$$

que l'on peut meltre sous la forme:

$S=\int_{B}^{C} \rho\left[1+\frac{1}{2}\left(\frac{d \rho}{\rho d 0}\right)^{2}-\frac{1}{8}\left(\frac{d \rho}{\rho d 0}\right)^{4} \cdots\right] d 0$ dans lacuelle nous avons:

$$
\begin{aligned}
& \varphi=\mathrm{R}-y \\
& \theta=x / \mathrm{R}
\end{aligned}
$$

La resolution de l'intégrale donne, avec une approximation tres suffisante:

$$
S=\frac{\pi \mathrm{R}}{n}-\frac{y_{0} \pi}{2 n}+\frac{y_{0}^{2} \pi n}{16 \mathrm{R}}
$$

La variation de longueur d'une demi-onde est done de:

$$
\Delta \mathrm{S}=\frac{\pi \mathrm{R}}{n}-\mathrm{S}=\frac{y_{0} \pi}{2 n}-\frac{y_{0}{ }^{2} \pi n}{16 \mathrm{R}}
$$

En ctudiant la fonction $\Delta S=f\left(y_{0}\right)$ (fig. 2), nous trouvons que $\Delta S$ est maximum pour :

$$
y_{i}=\frac{4 \mathrm{R}}{n^{2}}
$$

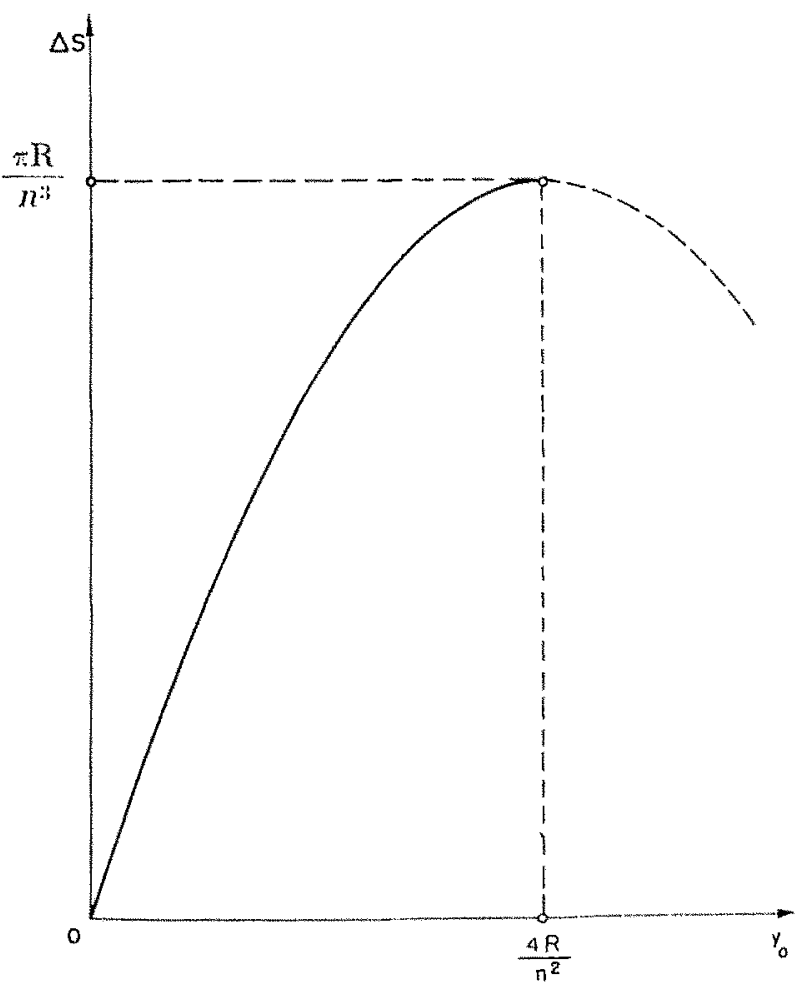

FIs. 2
Cette valeur correspond au flambage de londe puisque, au-delà, la longueur de l'arc BC aussmenle en même temps de $y_{0}$.

Portons celte valeur de $y_{0}$ dans l'équation (4), nous obtenons :

$$
\mathrm{M}_{r}=-\frac{\mathrm{E} e^{3}}{6 \mathrm{R}}
$$

Si nous appliquons celte formule a quelques cas concrets, nous nous rendons compte que cette valeur de $y_{0}$ ne peut pas être alteinte, car elle détermine, au point $C$, des contraintes de flexion qui dépassent de beaucoup la limite de rupture des aciers courants. II y aurait exception pour des blindages dont le rapport e/ $\mathrm{R}$ serait inférieur à $0,002 \div 0,003$. Ces blindages ne sont jamais utilisés pratiquement.

Dans un blindage soumis à une pression extérieure, la limite élastique ne doit être dépassíc en aucun point. Il est donc normal de calculer un blindage de telle façon qu'au point $C$, où la contrainte de flexion $t_{f}$ s'ajoute à une contrainte de compression $t^{\prime}$, la limite élastique $t$ ne soit pas dépassée :

$$
t_{f}+t_{t} \leqslant t
$$

Par suite des irrégularités d'épaisseur de la tôle et de qualité du métal, la limite élastique est atteinte en premier lieu dans une seule onde. A partir de cet instant, une augmentalion de la pression extérieure détermine, dans cette onde, une déformation permanente de grande amplitude par rapport aux déformations élastiques des ondes voisines, ce qui a pour eflet de rompre l'équilibre qui existe entre les ondes et de provoquer le flambage. D'autre part, il a été constaté, dans les quelques exemples de flambige connus, ainsi que dans les essais dont le compte rendu suit celte nole, que les tuyaux, après flambage, ne présentent qu'une onde de déformation sans que l'on puisse déceler un autre point où la limite élastique ail élé dépassée.

La condilion de llambage est done :

$$
t=\frac{\mathrm{E} e y_{0} n^{2}}{4 \mathrm{R}^{2}}+\frac{\mathrm{PR}}{e}
$$

Si $j$ est le jeu existant pour $P=0$, entre le cylindre et l'enveloppe, le développement de celleci est :

$$
2 \pi(\mathrm{R}+j)
$$

La longueur du cylindre sous la pression $P$ est :

$$
2 \pi R-2 \pi \frac{P R^{2}}{E \ell}
$$

Si $2 n \Delta S$ esl la dimérence de longueur entre 
l'enveloppe et le cylindre sous la pression P, nous avons :

$$
2 n \Delta \mathrm{S}=2 \pi\left(\frac{\mathrm{PR}^{2}}{\mathrm{E} e}+i\right)
$$

Les équations (5) et (7) donnent :

$$
\frac{y_{0}}{2}-\frac{y_{0}{ }^{2} n^{2}}{16 \mathrm{R}}=\frac{\mathrm{PR}^{2}}{\mathrm{E} e}+j
$$

Les équations (1), (6) et (8) permettent d'exprimer la pression de flambage $\mathrm{P}$ en fonction de $\mathrm{E}, j, t$ et $\mathrm{K}=(e / \mathrm{R})$.

P est la racine positive de l'équation :

$$
\frac{13}{\mathrm{EK}^{2}} \mathrm{P}^{2}+2 \mathrm{P}\left(1+\frac{\mathrm{K}}{2}+\frac{6}{\mathrm{~K}} \frac{j}{\mathrm{R}}-\frac{t}{\mathrm{EK}}\right)-\left(2 \ell \mathrm{K}-\mathrm{EK}^{2} \frac{j}{\mathrm{R}}-\frac{l^{2}}{\mathrm{E}}\right)=0
$$

\section{II. - APPLICATION AUX BLINDAGES}

L'équation (9 est applicable aux anneaux ou tubes courts. Pour l'utiliser sur des conduites longues (blindages métalliques de galeries et puits bétonnés) où la section longitudinale ne gauchit pas, il $\mathrm{y}$ a lieu de remplacer $\mathrm{E}$ par la valeur :

$$
E^{\prime}=\frac{E}{1-r_{1}^{2}}
$$

oì $\eta$ est le coefficient de Poisson.

Généralement, la pression extérieure admise pour les calculs de flambage est égale à la couverture de terrain $H$, située au-dessus de la conduite, qu'on affecte d'un coefficient de sécurité convenable. Si le blindage peut, accidentellement, être soumis au vide intérieur, il y a lieu d'en tenir compte et d'augmenter la valeur de $\mathrm{H}$ en conséquence.

Nous donnons à la figure 3 un graphique qui permet de trouver rapidement, pour une pression extérieure donnée, le $e / \mathrm{R}$ correspondant. Ce graphique a été établi avec $t=24$ et $34 \mathrm{~kg} / \mathrm{mm}^{2}$, et $\mathrm{J} / \mathrm{R}=0$ et 0,0004 . Cette dernière valeur représente le maximum de ce qu'on utilise pratiquement.

Nous avons également tracé les droites $\mathrm{P}=t(e / \mathrm{R})$ pour $t=24$ et 34 , qui donnent les pressions déterminant dans la tôle, s'il n'y avait pas flambage, une compression égale à la limite ćlastique. Nous constatons que les courbes donnant la pression de flambage se placent en dessous des droites correspondantes.

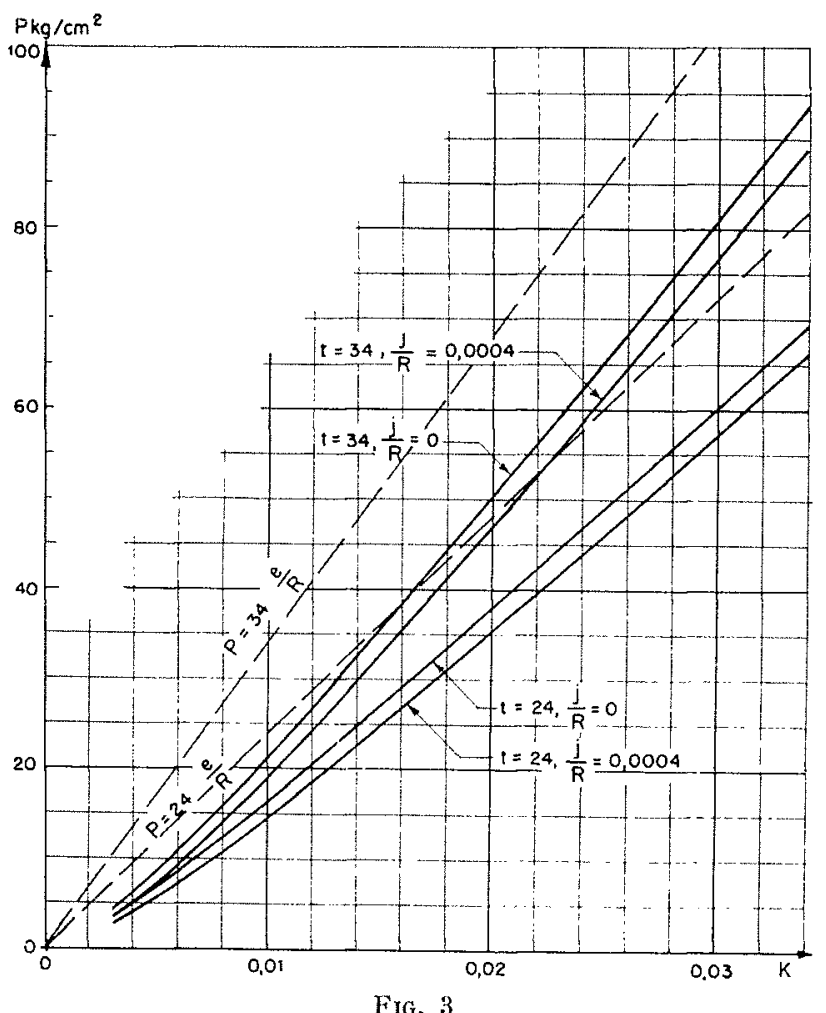

Courbes domnant les pressions de flambage $P$ en fonction de $\mathrm{K}=e / \mathrm{R}$, pour $t=24$ et $34 \mathrm{~kg} / \mathrm{mm}^{2}$ (.) $j / R=0$ at $0,0004 E^{\prime}=22.000 \mathrm{~kg} / \mathrm{mm}^{2}$.

\section{III. - VÉRIFICATION EXPÉRIMENTALE}

Lintérêt d'une formule se trouve augunentí quand celle-ci est vérifiće expćrimentalement. Des essais, effectués en laboratoire, nous ont permis de contrôler les chiffres donnés par la formule (9).

Nous avons construit un moule de $955,5 \mathrm{~mm}$ de diamètre intérieur (lig. 4) dans lequel ont été placćes des viroles dont l'épaisseur variait de 3,5 à 15 mm. La pression hydraulique a été appliquée entre le moule el la race extérieure de la tòle.

La limite élastique des tôles a été mesurée à 


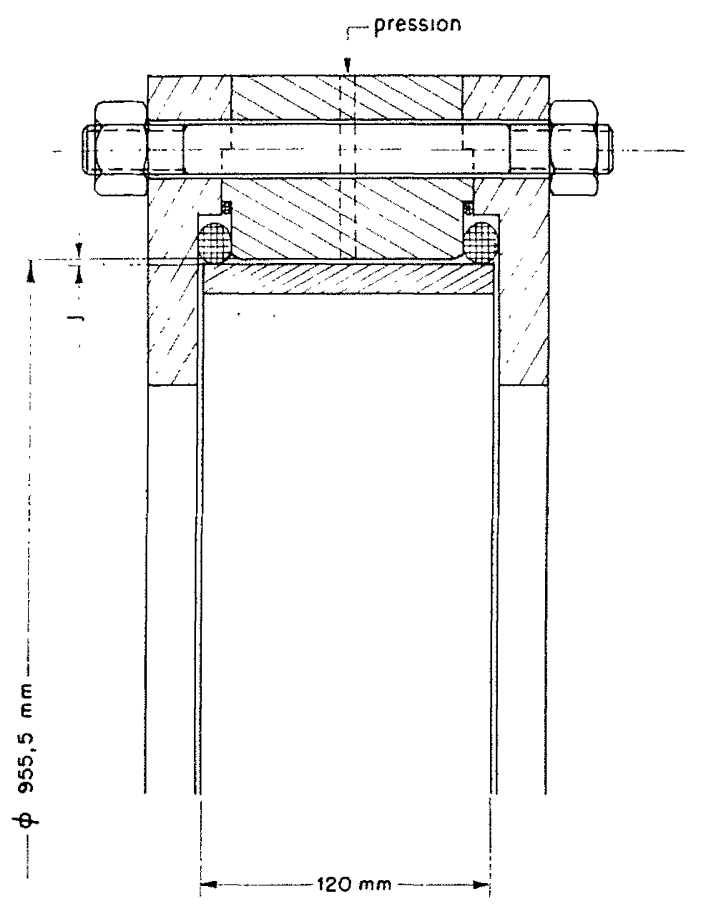

FIG. 4

Coupe de l'appareil pour essais de flambage.

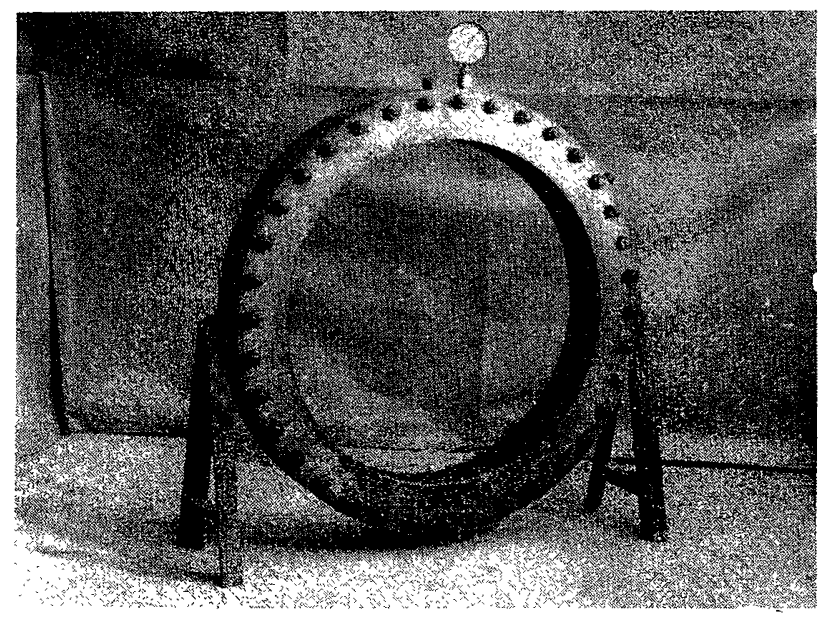

Pното 1. - Appareil pour essais de flambage.

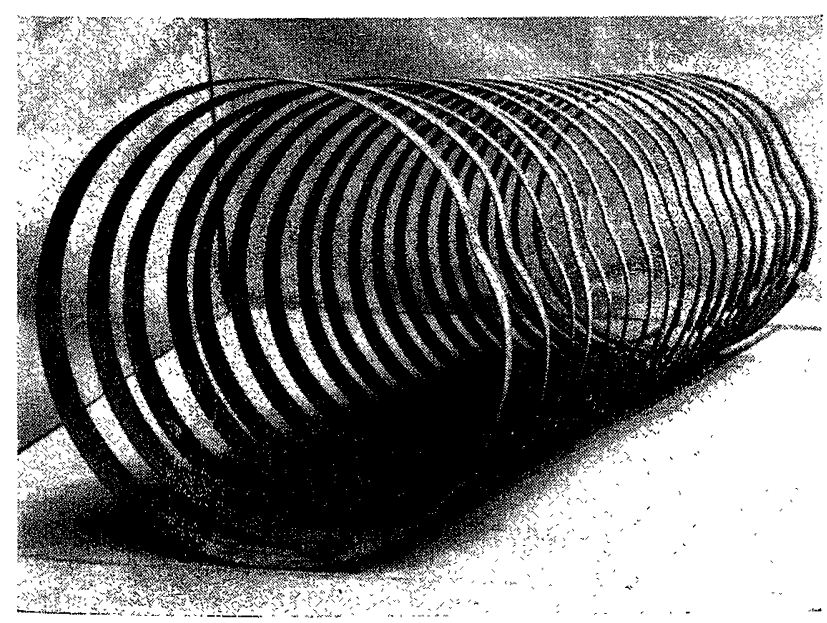

Photo 2. ... Viroles apres llambage. l'aide d'éprouvettes de $1,5 \mathrm{~mm}$ de diamètre, tractionnées sur une Micromachine Chevenard à enregistrement photographique. Le prélèvement des échantillons dans les viroles est visible sur les photographies.

Nous estimons à $\pm 0,3 \mathrm{~mm}$ la précision avec laquelle le jeu existant entre la tòle et le moule a été mesuré.

\section{'TABLEAU I}

RÉSUltats DES ESSAIS ET VÉRIFICATION PAR LA FORMULE (9)

\begin{tabular}{|c|c|c|c|c|c|}
\hline \multirow{2}{*}{ 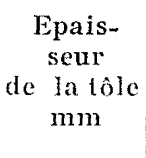 } & \multirow{2}{*}{$\begin{array}{c}\text { Jeu } \\
\text { (sur } \\
\text { rayon) } \\
\text { mm }\end{array}$} & \multirow{2}{*}{$\begin{array}{c}\text { Limite } \\
\text { elas- } \\
\text { tique } \\
\mathrm{kg} / \mathrm{mm}^{2}\end{array}$} & \multicolumn{2}{|c|}{$\begin{array}{c}\text { Pression de flambage } \\
\left(\mathrm{kg} / \mathrm{cm}^{2}\right)\end{array}$} & \multirow{2}{*}{$\begin{array}{l}\text { Diffé- } \\
\text { rence } \\
\text { en } \%\end{array}$} \\
\hline & & & Essais & $\underset{(9)}{\text { Formule }}$ & \\
\hline 3,5 & 1,1 & 27 & 4,8 & 5,8 & $-17,2$ \\
\hline 5 & 1,2 & 30 & 10 & 11 & $-9,1$ \\
\hline 5 & 0,9 & 31 & 11,5 & 12,9 & $-10,8$ \\
\hline 6 & 1 & 29 & 13,5 & 15,4 & $-12,3$ \\
\hline 6 & 0,6 & 29 & 20,5 & 18,3 & +12 \\
\hline 7 & 1 & 31 & 22,5 & 20,6 & $+9,2$ \\
\hline 7,2 & 1 & 30 & 21 & 20,9 & $+\quad 0,5$ \\
\hline 8,6 & 1,2 & 37 & 30,5 & 30,5 & 0 \\
\hline 9 & 0,9 & 33 & 32 & 32,3 & $-\quad 0,9$ \\
\hline 9 & 1 & 25 & 28 & 24,4 & $+14,8$ \\
\hline 9,8 & 1,1 & 34 & 35 & 35,2 & $-0,6$ \\
\hline 10,9 & 0,6 & 33 & 49 & 46,2 & $+6,1$ \\
\hline 11,3 & 1,2 & 38 & 51 & 46,2 & $+10,4$ \\
\hline 12 & 1,1 & 34 & 48 & 46,7 & $+2,8$ \\
\hline 12,9 & 1,1 & 37 & 62 & 55,7 & $+11,3$ \\
\hline 13,6 & 0,6 & 25 & 55 & 48,1 & $+14,3$ \\
\hline 14 & 0,5 & 27 & 60 & 55 & $+9,1$ \\
\hline 14,7 & 0,5 & 33 & 77 & 69,7 & $+10,5$ \\
\hline 15 & 1,2 & 34 & 63 & 61,7 & $+2,1$ \\
\hline
\end{tabular}

Les résultats des essais sont consignés dans le tableau 1. Sur ce tableau figurent également les pressions de flambage données par la formule (9) pour chaque virole, ainsi que la différence en pourcentage entre l'essai et le calcul.

Celle différence est d'environ $=15 \%$, et si nous tenons compte du manque de précision de la valeur du jeu, nous pouvons dire que la formule (9) n'a pas élé mise en défaut.

Nous avons porté les résultats des essais sur des graphiques (fig. 5, 6, 7 et 8 ) où figurent des courbes qui donnent, pour un diamètre nominal de $955 \mathrm{~mm}$, les pressions de flambage trouvées par la formule (9), en fonction de l'épaisseur des viroles. 


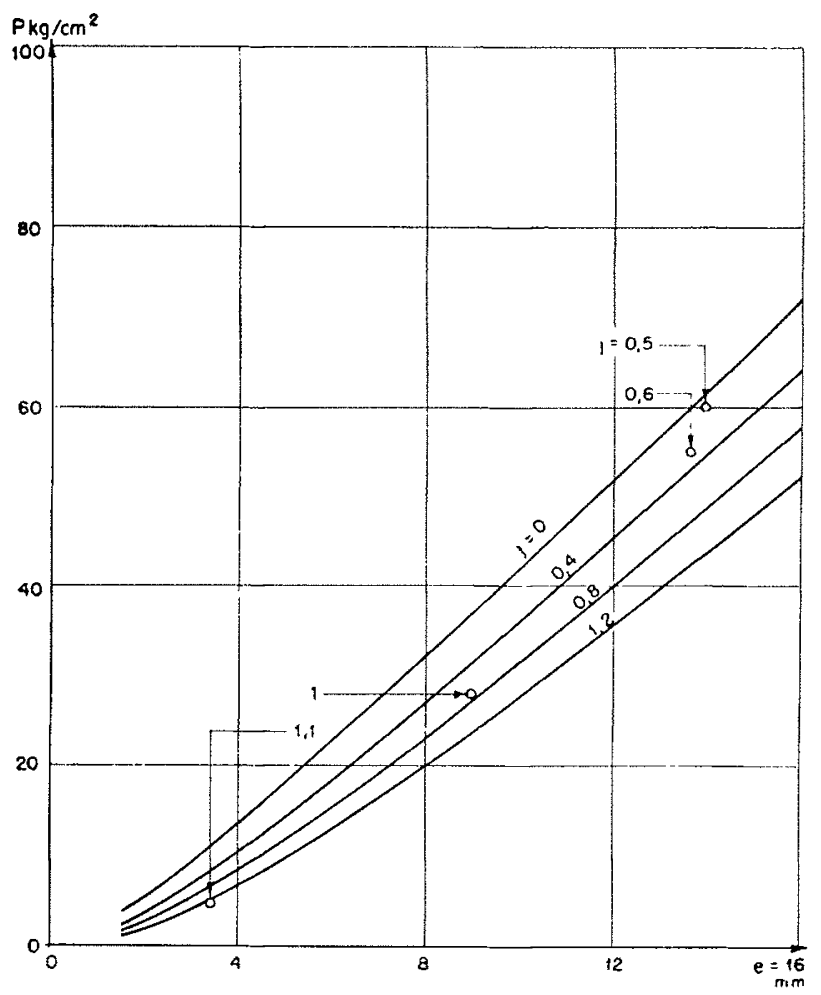

FIG. 5

Dispersion des résultats d'essais : $t=26 \mathrm{~kg} / \mathrm{mm}^{2}$

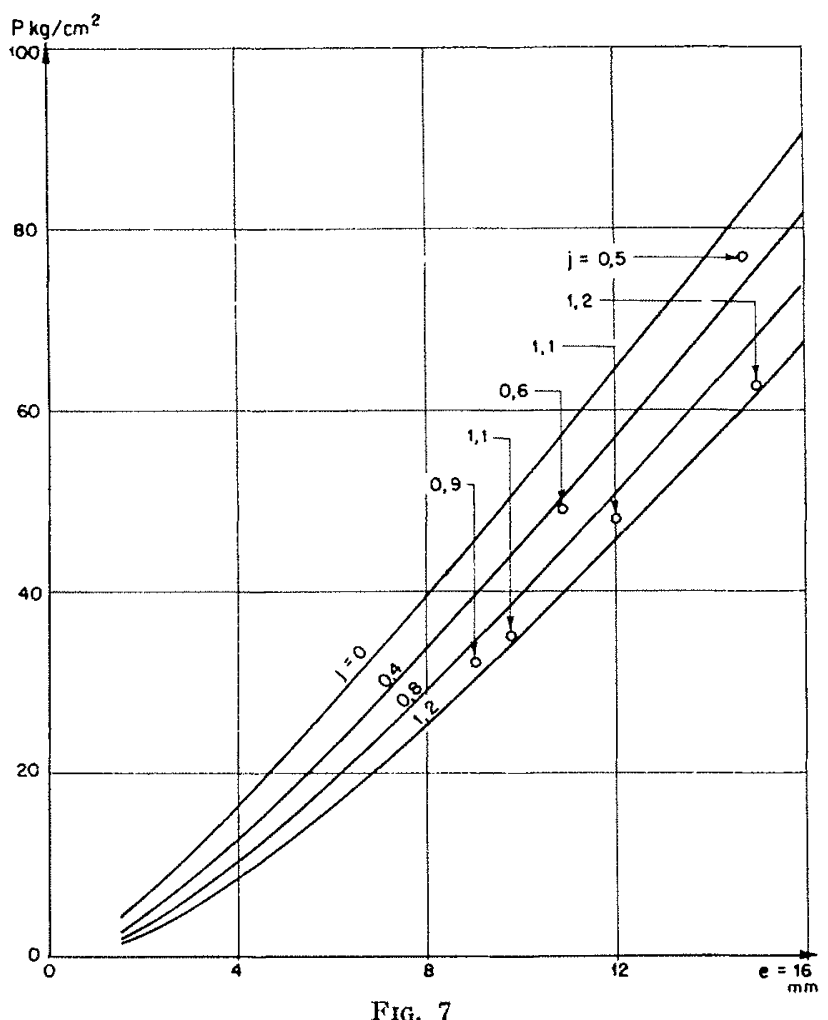

Dispersion des résultats d'essais : $t=34 \mathrm{~kg} / \mathrm{mm}^{2}$

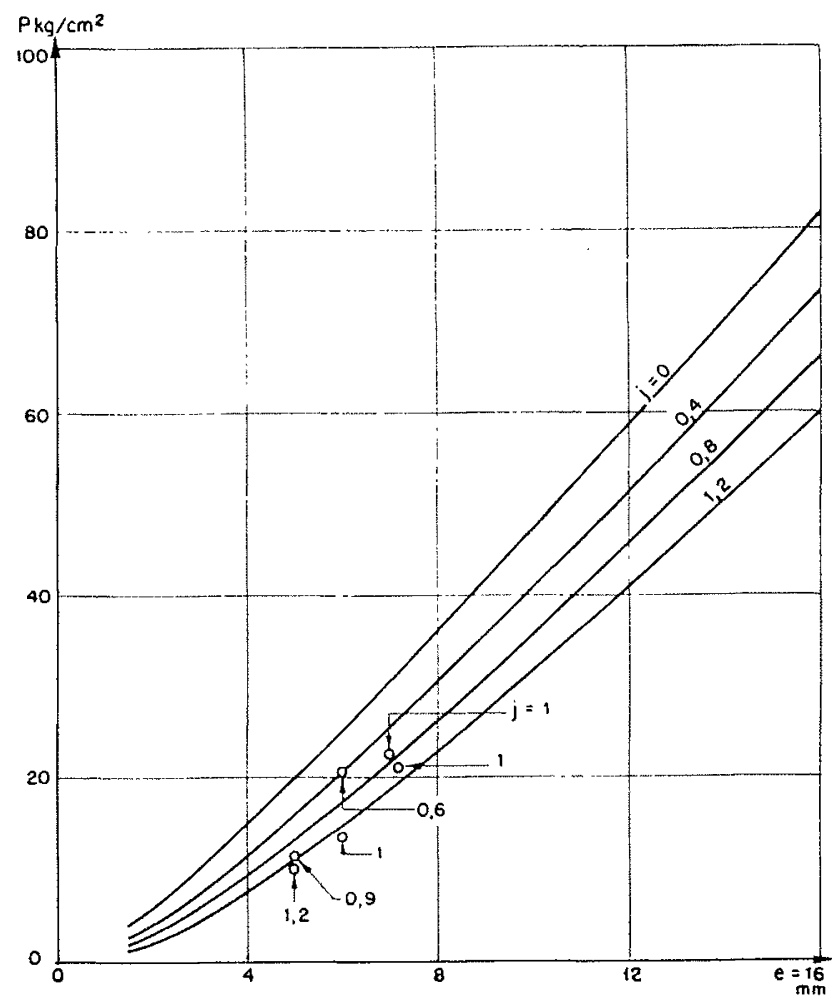

FIG. 6

Dispersion des résultats d'essais : $t=30 \mathrm{~kg} / \mathrm{mm}^{2}$

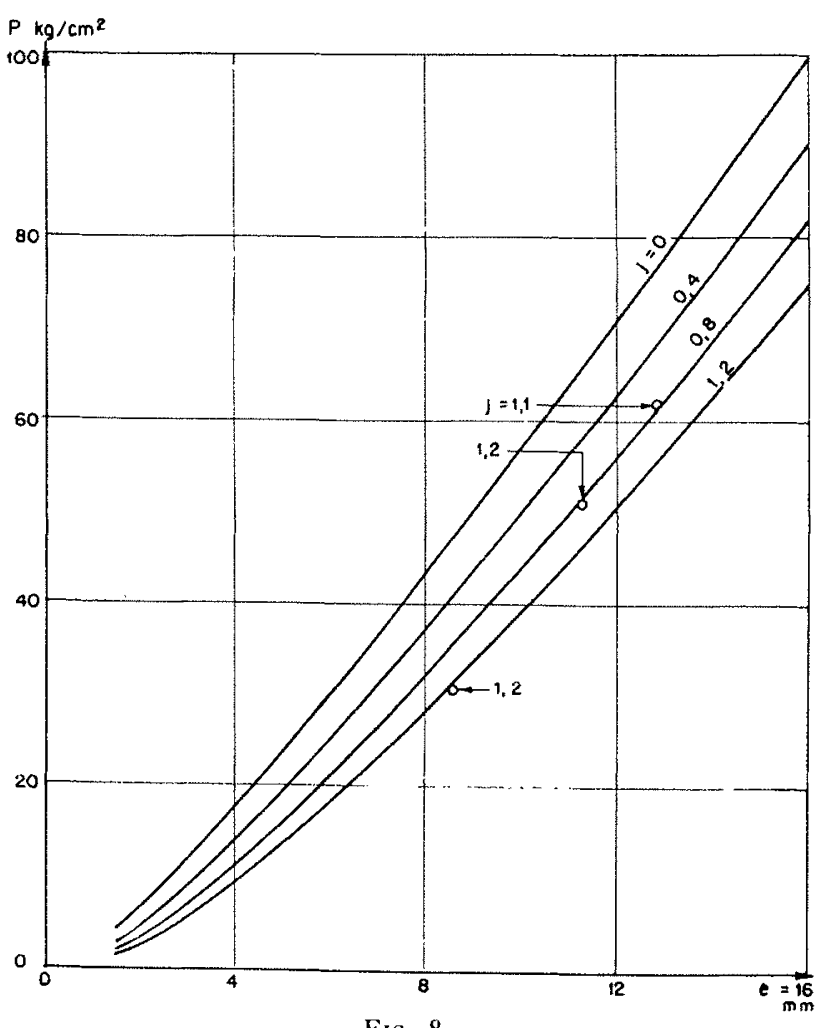

FIG. 8

Dispersion rles résultats d'essais : $t=38 \mathrm{~kg} / \mathrm{mm}^{2}$ 
Nous avons groupé :

- sur la figure 5 , les viroles dont la limite ćlastique était de 25 à $27 \mathrm{~kg} / \mathrm{mm}^{2}$;

- sur la figure 6 , de 29 à $31 \mathrm{~kg} / \mathrm{mm}^{2}$;

... sur la figure 7 , de 33 et $34 \mathrm{~kg} / \mathrm{mm}^{2}$;

-.. sur la figure 8 , de 37 et $38 \mathrm{~kg} / \mathrm{mm}^{2}$.
Les courbes des graphiques sonl tracées respectivement pour :

$$
t=26,30,34 \text { et } 38 \mathrm{~kg} / \mathrm{mm}^{2}
$$

el pour :

$$
j=0,0,4,0,8 \text { et } 1,2 \mathrm{~mm}
$$

Les viroles ont une longueur de $120 \mathrm{~mm}$; nous les avons assimilées à des anneaux et nous avons calculé les courbes avec $\mathrm{E}=20000 \mathrm{~kg} / \mathrm{mm}^{2}$.

\section{CONCLUSION}

La formule (9) permet done de dimensionner les blindages non précontraints de puits ou galerie, qui sont sollicités aux pressions extérieures, en prenant soin d'être prudent dans la détermination de ces dernières.

Si cette formule a eu l'avantage d'avoir été vérifiée par des essais, il n'en serait pas moins intéressant de l'appliquer à des cas concrets pour lesquels la limite élastique du hlindage et la valeur de la pression extérieure ayant provoqué le flambage sont connues.

Celte étude met bien en évidence l'influence de la limite élastique sur la résistance au flambage et montre clairement l'intérêt de l'emploi d'acier à haute limite élastique qui donne aux blindages, une meilleure aptitude à résister aux pressions extérieures.

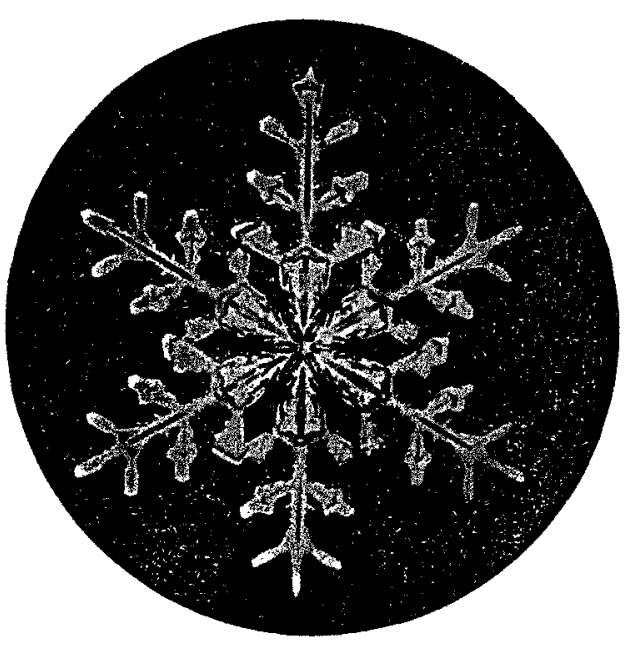

